\title{
Wasserstein metric-driven Bayesian inversion with applications to signal processing
}

\author{
M. Motamed ${ }^{* 1}$ and D. Appelö ${ }^{\dagger 2}$ \\ ${ }^{1}$ Department of Mathematics and Statistics, University of New Mexico, USA \\ ${ }^{2}$ Department of Applied Mathematics, University of Colorado Boulder, USA
}

December 31, 2018

\begin{abstract}
We present a Bayesian framework based on a new exponential likelihood function driven by the quadratic Wasserstein metric. Compared to conventional Bayesian models based on Gaussian likelihood functions driven by the least-squares norm $\left(L_{2}\right.$ norm), the new framework features several advantages. First, the new framework does not rely on the likelihood of the measurement noise and hence can treat complicated noise structures such as combined additive and multiplicative noise. Secondly, unlike the normal likelihood function, the Wasserstein-based exponential likelihood function does not usually generate multiple local extrema. As a result, the new framework features better convergence to correct posteriors when a Markov Chain Monte Carlo sampling algorithm is employed. Thirdly, in the particular case of signal processing problems, while a normal likelihood function measures only the amplitude differences between the observed and simulated signals, the new likelihood function can capture both amplitude and phase differences. We apply the new framework to a class of signal processing problems, that is, the inverse uncertainty quantification of waveforms, and demonstrate its advantages compared to Bayesian models with normal likelihood functions.
\end{abstract}

Keywords. Bayesian inversion; Wasserstein metric; measurement noise; Markov Chain Monte Carlo; Metropolis-within-Gibbs Sampling; signal processing; uncertainty quantification; waveforms

MSC. 62F15, 62P30, 65M32, 60J22, 86A15, 35L53

\section{Introduction}

The Euclidean distance is one of the most widely used metrics in constructing misfit functions in deterministic inversion algorithms. In a Bayesian framework (see e.g. [14, 18, 30]), this misfit function corresponds to a normal likelihood function, which is valid under the (often unrealistic) assumption that the experimental noise is additive and Gaussian. In addition to being unrealistic, a Gaussian likelihood function measures only the amplitude differences between the observed and simulated signals and does not capture the phase differences.

*motamed@math.unm.edu

†daniel.appelo@colorado.edu 
Moreover, Gaussian likelihood functions often have multiple local extrema that may result in converging to wrong posteriors (see Section 5) when a Markov Chain Monte Carlo (MCMC) sampling algorithm [13] is used. Such disadvantages are particularly of great concern in signal processing applications, such as seismic inversion problems, where the noise is not necessarily additive and the wave signals are oscillatory and cyclic.

In the present work we will propose a Bayesian framework based on an exponential likelihood function driven by the quadratic Wasserstein metric; see e.g. [31, 32]. We will compare the new framework with current Bayesian models based on normal likelihood functions when applied to a class of signal processing problems, including seismic source inversion and seismic imaging. In particular the ideas presented here find their inspiration in the recent activity in the area of (deterministic) Full Waveform Inversion (FWI) exploiting distances connected to optimal transportation. The use of the quadratic Wasserstein distance in FWI is relatively new but has had substantial impact in the exploration community as well as in the mathematical geosciences community. Engquist and Froese first proposed to use the quadratic Wasserstein distance for seismic signals in [11]. This work has been analyzed and extended in a sequence of papers by Yang, Engquist, and co-authors [9, 33, 10] clearly demonstrating the power of the approach when applied to FWI. We note that the quadratic Wasserstein metric has recently been used to invert for source mechanisms and location in [6, 2].

We further note that in FWI there is also considerable interest in the approach by Métivier and co-authors, [23, 24, 21, 22, 17]. In their approach the quadratic Wasserstein distance is replaced by a relaxed 1-Wasserstein distance obtained from the Monge-Kantorovich problem via a maximization in the space of bounded 1-Lipschitz functions. Unlike the quadratic Wasserstein distance the resulting distance does not rely on normalizing the input signals to be positive and with equal mass.

Given the success of both approaches in FWI, a natural extension of this work would be to use the techniques from [23, 24, 21, 22] in place of the quadratic Wasserstein distance.

In this paper we focus on the quadratic Wasserstein and extend the work [11, 9, 33, 10] to Bayesian inversion and demonstrate applications of the new framework in the uncertainty quantification of seismic inversion problems.

The rest of the paper is organized as follows. In Section 2 we formulate the Bayesian inversion problem and discuss the likelihood and measurement noise structures. We then present the new Wasserstein metric-driven Bayesian framework in Section 3, followed by a standard MCMC algorithm in Section 4. We finally present several numerical examples related to seismic wave inversion in Section 5 .

\section{Bayesian inversion problem}

\subsection{Problem formulation}

Let $\mathbf{g}=\left(g_{1}, \ldots, g_{N}\right) \in \mathbb{R}^{N}$ be a vector of $N$ observed quantities, for instance, measured by an experimental device. Let further $\mathbf{f}=\left(f_{1}, \ldots, f_{N}\right) \in \mathbb{R}^{N}$ be a vector of $N$ predicted quantities corresponding to $\mathbf{g}$, computed by a forward predictive model with an input parameter vector $\boldsymbol{\theta}=\left(\theta_{1}, \ldots, \theta_{m}\right) \in \Theta \subset \mathbb{R}^{m}$ :

$$
\mathbf{f}=\mathbf{f}(\boldsymbol{\theta}): \Theta \subset \mathbb{R}^{m} \rightarrow \mathbb{R}^{N}
$$


A main assumption in mathematical statistics is that the model parameter vector $\boldsymbol{\theta}$, and consequently any physical quantity to be predicted by the forward model, is described by a stochastic law characterized by a probability density function (PDF). The density of $\boldsymbol{\theta}$ is available to us only through the experimental measurements $\mathbf{g}$ that are often corrupted by experimental noise and error. The goal is then to find the density of $\boldsymbol{\theta}$, given the forward model $\mathbf{f}(\boldsymbol{\theta})$ and the noisy data $\mathbf{g}$.

In Bayesian inversion the conditional posterior probability density of the model parameter vector $\boldsymbol{\theta}$ is given by the Bayes' rule; see e.g. [3, 14, 18, 30]:

$$
\pi(\boldsymbol{\theta} \mid \mathbf{g})=\frac{\pi(\mathbf{g} \mid \boldsymbol{\theta}) \pi(\boldsymbol{\theta})}{\int_{\Theta} \pi(\mathbf{g} \mid \boldsymbol{\theta}) \pi(\boldsymbol{\theta}) d \boldsymbol{\theta}} \propto \pi(\mathbf{g} \mid \boldsymbol{\theta}) \pi(\boldsymbol{\theta}),
$$

where $\pi(\mathbf{g} \mid \boldsymbol{\theta})$ is the likelihood, and $\pi(\boldsymbol{\theta})$ is the prior density of $\boldsymbol{\theta}$. The proportionality follows from the fact that the denominator (referred to as the evidence) does not depend on $\boldsymbol{\theta}$ and hence can be regarded as a constant. Assuming the observed samples $\left\{g_{i}\right\}_{i=1}^{N}$ are independent, their joint likelihood reads

$$
L(\boldsymbol{\theta}):=\pi(\mathbf{g} \mid \boldsymbol{\theta})=\prod_{i=1}^{N} \pi\left(g_{i} \mid \boldsymbol{\theta}\right),
$$

where the first equality indicates that the likelihood can be viewed as a function of only $\boldsymbol{\theta}$, referred to as the likelihood function and denoted by $L(\boldsymbol{\theta})$.

\subsection{Likelihood and noise structure}

A major step in Bayesian analysis is the selection of the likelihood function. This is usually done based on the structure of the measurement noise. The most common choice is the Gaussian likelihood, built based on the assumption that the measurement noise $\left\{\varepsilon_{i}\right\}_{i=1}^{N}$ in the $N$ measured quantities $\left\{g_{i}\right\}_{i=1}^{N}$ is additive and normally distributed with zero mean and a standard deviation $\sigma$ :

$$
g_{i}=f_{i}(\boldsymbol{\theta})+\varepsilon_{i}, \quad \varepsilon_{i} \sim \operatorname{Normal}(0, \sigma), \quad i=1, \ldots, N .
$$

In this case the likelihood function reads

$$
L_{\text {norm }}(\boldsymbol{\theta})=\pi_{\text {norm }}(\mathbf{g} \mid \boldsymbol{\theta})=\frac{1}{(2 \pi)^{N / 2} \sigma^{N}} \exp \left(\frac{-1}{2 \sigma^{2}} \sum_{i=1}^{N}\left|g_{i}-f_{i}(\boldsymbol{\theta})\right|^{2}\right) .
$$

One critical problem with this setting is that the Gaussian additive noise assumption may not be realistic. For instance, in ultrasound and laser imaging with $\mathbf{g} \in \mathbb{R}^{N}$ being an $N$-pixel image, the noise may be multiplicative. A practical example is a speckle noise (see e.g. [15]) with mean one and variance $1 / s$ :

$$
g_{i}=\varepsilon_{i} f_{i}(\boldsymbol{\theta}), \quad \varepsilon_{i} \sim \operatorname{Gamma}(s, 1 / s), \quad i=1, \ldots, N .
$$

Here $\operatorname{Gamma}(a, b)$ is a Gamma distribution with a shape parameter $a>0$ and a rate parameter $b>0$. In some applications, the noise structure may be even more complicated. For instance the measurement noise may be both additive and multiplicative, taking the form

$$
g_{i}=\varepsilon_{i}^{(1)} f_{i}(\boldsymbol{\theta})+\varepsilon_{i}^{(2)}, \quad i=1, \ldots, N .
$$


Such complicated noise structures may not be easily treated by Bayesian frameworks that rely on the likelihood of the noise, even when a non-Gaussian likelihood function is employed. It is to be noted that the noise structure is not the only problem with Gaussian likelihoods. Even if the noise structure is additive and can be modeled by a Gaussian likelihood function, the likelihood may have multiple local extrema, resulting in wrong posteriors when applying a MCMC sampling method; see Section 5. Moreover, in signal processing problems, a Gaussian likelihood function that is based on the $L_{2}$ norm measures only the amplitude differences between the observed and predicted signals and does not capture the phase differences. This latter problem is indeed a crucial limitation for instance in: a) seismic inversion with moderately to highly oscillatory seismic waves, and b) MRI noise reduction where the retainment of the image fine features is desired.

In what follows we present a Bayesian framework based on an exponential likelihood function driven by a quadratic Wasserstein metric. Unlike conventional Bayesian analysis, this framework does not rely on the likelihood of the measurement noise and hence can treat complicated noise structures as in (1). Moreover, since the Wasserstein metric has better optimization and fitting properties than the least-squares norm (see e.g. [33] and the references therein), the new framework may feature better convergence properties than the standard frameworks based on Gaussian likelihoods.

\section{Wasserstein metric-driven Bayesian framework}

The Wasserstein metric is a distance function defined between two probability distributions. It corresponds to the minimum "cost" of turning one distribution into the other. The metric has been applied to various fields, including image processing [28], computer vision [19], Stochastic programming, [12], and seismic imaging [33]. We refer to [31, 32] for a complete introduction to Wasserstein distances.

Consider two discrete-time signals $\mathbf{f}, \mathbf{g} \in \mathbb{R}^{N}$ given at a discrete set of time level $\left\{t_{i}\right\}_{i=1}^{N}$. Since the two (oscillatory) signals are not necessarily probability distributions, i.e. positivity and mass balance are not expected, the signals need to be pre-processed before we can measure their Wasserstein distance. Here, we follow the normalization via a linear transformation and rescaling proposed in [33] and construct two discrete CDFs as follows:

- Shift the signals to ensure positivity: select a constant $c$ so that $f_{i}+c>0$ and $g_{i}+c>0$, for all $i=1, \ldots, N$.

- Rescale the shifted signals so that they share a common unit total mass:

$$
\tilde{\mathbf{f}}=\frac{\mathbf{f}+c}{\langle\mathbf{f}+c\rangle}, \quad \tilde{\mathbf{g}}=\frac{\mathbf{g}+c}{\langle\mathbf{g}+c\rangle},
$$

where $\langle\mathbf{f}\rangle=\sum_{i=1}^{N} f_{i}$ is the total mass of the signal $\mathbf{f}$.

- Find the discrete CDFs $F=\left(F_{1}, \ldots, F_{N}\right)$ and $G=\left(G_{1}, \ldots, G_{N}\right)$ with the components

$$
F_{i}=\sum_{j=1}^{i} \tilde{f}_{j}, \quad G_{i}=\sum_{j=1}^{i} \tilde{g}_{j}, \quad i=1, \ldots, N
$$


where $\tilde{f}_{j}$ and $\tilde{g}_{j}$ are the $j$-th components of $\tilde{\mathbf{f}}$ and $\tilde{\mathbf{g}}$ in (2), respectively.

The discrete quadratic Wasserstein distance between the two signals then reads

$$
d_{W}(\mathbf{f}, \mathbf{g})=\sum_{i=1}^{N}\left|t_{i}-T_{i}\right|^{2} \tilde{f}_{i}, \quad T=G^{-1} \circ F .
$$

We note that $T=G^{-1} \circ F$ is the "optimal" map from the density $\tilde{\mathbf{f}}$ to the density $\tilde{\mathbf{g}}$. Intuitively, if we view the two densities as two piles of dirt with the same unit mass, the map would correspond to the minimum "cost" of turning one pile into the other, i.e. the minimum amount of dirt that needs to be moved times the distance it has to be moved. This corresponds to the horizontal distance between the two discrete CDFs $F$ and $G$. For computing the metric $d_{W}$ in (4), the map $T$ is computed by interpolation.

We then consider an exponential likelihood function

$$
L_{\exp }(\boldsymbol{\theta})=\pi_{\exp }(\mathbf{g} \mid \boldsymbol{\theta})=s^{N} \exp \left(-s d_{W}(\mathbf{f}(\boldsymbol{\theta}), \mathbf{g})\right)
$$

where $s>0$ is a rate parameter and will be considered as a hyperparmeter in the MCMC sampling algorithm. The main motivation for the selection of an exponential function is that we would like to recover the Wasserstein-driven cost functional in deterministic inverse problems by taking the negative logarithm of the posterior in the Bayesian setting [30].

It is to be noted that the Wasserstein metric can be computed in any dimension through the (numerical) solution of an Monge-Ampère equation, see e.g. [4]. For dimensions larger than one, where there are more than one signal, this may be a costly procedure. We therefore follow the work by Yang et. al. [33] and use a "trace-by-trace" approach, summing up one dimensional quadratic Wasserstein distances; see Section 5 where there are more than one signal collected by several receivers.

Convexity of the Wasserstein exponential likelihood. One important feature of the quadratic Wasserstein metric is its convexity with respect to the phase shift, phase dilation, and amplitude change in the simulated and measured signals [9]. However, due to normalization during the pre-processing step, the convexity may be lost. This may in turn lead to the cycle skipping issue in the proposed Bayesian framework, i.e. the presence of multiple local optima in the exponential likelihood function (5). We notice that various normalization strategies, in the context of FWI, have been proposed to overcome this issue; see e.g. [11, 9, 33]. To see the effect of different normalization strategies on the convexity of the quadratic Wasserstein distance with respect to phase shifts and to compare the cycle skipping issue with that of the usual $L_{2}$ distance, we consider a simple example with two identical but shifted signals. Precisely the original signal $f$ is

$$
f(t)=e^{-\left(\frac{t-4}{\delta}\right)^{2}}-e^{-\left(\frac{t-5}{\delta}\right)^{2}}+e^{-\left(\frac{t-6}{\delta}\right)^{2}},
$$

and $g$ is a shifted version of $f$

$$
g(t)=e^{-\left(\frac{t-s-4}{\delta}\right)^{2}}-e^{-\left(\frac{t-s-5}{\delta}\right)^{2}}+e^{-\left(\frac{t-s-6}{\delta}\right)^{2}}
$$



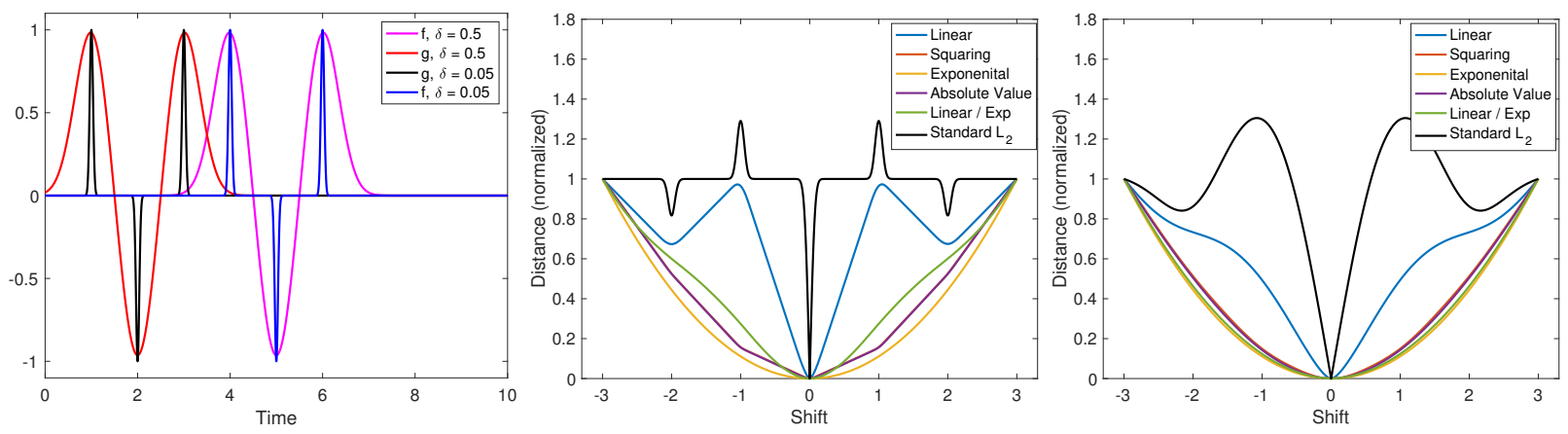

Figure 1: Comparison of different normalization / scaling procedures for the quadratic Wasserstein distance and the standard $L_{2}$ distance for two signals with different width (see the text for descriptions of the legend). The middle figure is for the narrow signals and the right is for the wide signals.

with shift $s$. In Figure 1 we compare the convexity of the distance between $f$ and $g$ as a function of the shift and for different normalizations. The values of the distances have been scaled to start at one when the shift is -3 . The different options to preserve positivity we use are

$$
\begin{array}{cl}
\hat{f}=f+c, & \text { Linear scaling, } \\
\hat{f}=f^{2}, & \text { Square scaling, } \\
\hat{f}=\exp (c f), & \text { Exponential scaling, } \\
\hat{f}=|f|, & \text { Absolute value scaling, } \\
\hat{f}=\left\{\begin{array}{cl}
\exp (c f) \text { if } f<0, & \text { Linear/Exponential scaling, } \\
f+1 / c \text { if } f \geq 0, &
\end{array}\right.
\end{array}
$$

The generic constant $c$ should be chosen big enough to ensure positivity for the linear scaling and small enough for exponential scaling not to overflow.

The results in Figure 1 show that, in general, one should not expect to preserve the convexity of the quadratic Wasserstein distance when the linear scaling is used, as has been discussed recently in e.g. [10] and [22]. However, if the signals are not clearly separated (corresponding to a larger $\delta$ ) the linear scaling does preserve the convexity, see the rightmost subfigure. Compared to the $L_{2}$ distance the basin of attraction is significantly expanded even in the case with local minima. The other alternatives appears to give quite good results in terms of convexity, but it should be pointed out that the squaring and absolute value functions are not unique, in the sense that many signals $f$ can produce $\hat{f}$ and that these options are therefore typically not used in deterministic problems. The most robust option is probably the combination of the exponential and linear procedure.

We note that in all our computations below we use the linear scaling. For the one dimensional examples the main signals are positive but the added noise can make them slightly negative, activating the normalization procedure. However as the size of the noise is small we expect that the linear scaling results in a convex cost function. In the two 
dimensional examples below the discrete signals typically contain equal parts of positive and negative entries so that we cannot exclude the possibility of local minima. We plan to carry out a detailed exploration of the impact of the scaling on the proposed method in the future.

\section{Numerical algorithm}

We employ a Metropolis-Hastings-within-Gibbs sampling strategy (see e.g. [14]) within the proposed Bayesian framework. The algorithm, summarized in Algorithm 1, iteratively generates a sequence of samples, forming a Markov chain, whose distribution approaches the target distribution of parameters in the limit. A candidate sample is produced based on the current sample value and is later accepted or rejected with some probability $\alpha$; see below. The algorithm consists of two interactive parts: a Gibbs sampler and a Metropolis-Hastings sampler. The Gibbs sampler is used to sample the hyperparameter $s>0$ with a fixed parameter vector $\boldsymbol{\theta}$, and the Metropolis-Hastings sampler is used to sample $\boldsymbol{\theta}$ with a fixed hyperparameter $s$.

Gibbs sampler. The Gibbs sampler employs the proposed exponential likelihood (5) and a Gamma prior on its rate parameter $s$, i.e. $s \sim \operatorname{Gamma}(a, b)$, where $a>0$ and $b>0$ are the shape and rate parameters of the prior. Given a fixed $\boldsymbol{\theta}$ we will then have

$$
\pi(s \mid \boldsymbol{\theta}, \mathbf{g}) \propto \pi_{\exp }(\mathbf{g} \mid \boldsymbol{\theta}, s) \pi_{\text {prior }}(s) \propto s^{N} e^{-s d_{W}} s^{a-1} e^{-b s}=s^{a+N-1} e^{-s\left(b+d_{W}\right)},
$$

where $d_{W}=d_{W}(\mathbf{f}(\boldsymbol{\theta}), \mathbf{g})$ is the Wasserstein metric given by (4). Hence we obtain a Gamma posterior on $s$ :

$$
s \sim \operatorname{Gamma}\left(a^{*}, b^{*}\right), \quad a^{*}=a+N, \quad b^{*}=b+d_{W} .
$$

This will be used to generate a new sample of $s$, given a fixed $\boldsymbol{\theta}$.

The Gamma prior used here is a conjugate prior, giving a Gamma posterior for $s$. We notice that other priors may be used for the rate parameter $s$. Here, we use a Gamma prior, since the rate parameter is related to the precision parameter in a Gaussian likelihood, for which a Gamma prior is commonly used in practice. Of course, the change of prior of $s$ will change the posterior of $s$.

Metropolis-Hastings sampler. The Metropolis-Hastings sampler [25, 16] employs the proposed exponential likelihood (5) with a fixed rate parameter $s$ obtained by the Gibbs sampler. The posterior from which a sequence of samples are to be generated reads

$$
\pi(\boldsymbol{\theta} \mid s, \mathbf{g}) \propto \pi_{\exp }(\mathbf{g} \mid \boldsymbol{\theta}, s) \pi_{\text {prior }}(\boldsymbol{\theta})
$$

Given a sample value $\boldsymbol{\theta}^{(i)}$, a new sample $\boldsymbol{\theta}^{(i+1)}$ is generated as follows. A candidate sample, say $\tilde{\boldsymbol{\theta}}$, is first generated by a proposal distribution $q\left(\boldsymbol{\theta}^{(i)}, \tilde{\boldsymbol{\theta}}\right)$ from the current sample $\boldsymbol{\theta}^{(i)}$. This candidate sample is then accepted or rejected with probability (see e.g. [7])

$$
\alpha=\frac{\pi(\tilde{\boldsymbol{\theta}} \mid s, \mathbf{g}) q\left(\boldsymbol{\theta}^{(i)}, \tilde{\boldsymbol{\theta}}\right)}{\pi\left(\boldsymbol{\theta}^{(i)} \mid s, \mathbf{g}\right) q\left(\tilde{\boldsymbol{\theta}}, \boldsymbol{\theta}^{(i)}\right)}=\frac{\pi_{\exp }(\mathbf{g} \mid \tilde{\boldsymbol{\theta}}, s) \pi_{\text {prior }}(\tilde{\boldsymbol{\theta}}) q\left(\boldsymbol{\theta}^{(i)}, \tilde{\boldsymbol{\theta}}\right)}{\pi_{\exp }\left(\mathbf{g} \mid \boldsymbol{\theta}^{(i)}, s\right) \pi_{\text {prior }}\left(\boldsymbol{\theta}^{(i)}\right) q\left(\tilde{\boldsymbol{\theta}}, \boldsymbol{\theta}^{(i)}\right)}
$$


It is to be noted that the choice of prior would depend on the problem at hand and expert's opinion. For instance, the prior may be informative with uniform or other types of distributions, or it may be non-informative, i.e. $\pi_{\text {prior }}(\boldsymbol{\theta})=1$. We may even consider hierarchical priors that include other hyper-priors. For the sake of simplicity and without imposing any restriction, in the numerical examples in Section 5 we consider uniform priors on $\boldsymbol{\theta}$,

$$
\pi_{\text {prior }}(\boldsymbol{\theta})=\prod_{i=1}^{m} \pi_{\text {prior }}\left(\theta_{i}\right), \quad \theta_{i} \sim \operatorname{Unif}\left(\alpha_{i}, \beta_{i}\right),
$$

where the bounds $\left\{\left(\alpha_{i}, \beta_{i}\right)\right\}_{i=1}^{m}$ are to be selected depending on the problem at hand. We further use a Gaussian random walk proposal to generate a new sample $\tilde{\boldsymbol{\theta}}$ from a current sample $\boldsymbol{\theta}^{(i)}$ :

$$
\tilde{\boldsymbol{\theta}} \sim \operatorname{Normal}\left(\boldsymbol{\theta}^{(i)}, \Sigma\right)
$$

where $\Sigma \in \mathbb{R}^{m \times m}$ is a covariance matrix to be selected so that the proposal distribution is neither too wide nor too narrow. The former would result in an acceptance rate close to zero, and the chain would rarely move to a different sample. The latter would result in an acceptance rate close to one, however, the generated samples would not cover the whole support of posterior. We note that since such a proposal is symmetric, i.e. $q\left(\boldsymbol{\theta}^{(i)}, \tilde{\boldsymbol{\theta}}\right)=$ $q\left(\tilde{\boldsymbol{\theta}}, \boldsymbol{\theta}^{(i)}\right)$, the ratio $\alpha$ simplifies to $\alpha=\pi(\tilde{\boldsymbol{\theta}} \mid s, \mathbf{g}) / \pi\left(\boldsymbol{\theta}^{(i)} \mid s, \mathbf{g}\right)$. This ratio was used in the original version of the Metropolis algorithm [25].

Algorithm 1 Metropolis-Hastings-within-Gibbs Sampling in the Wasserstein-Bayesian framework

1. Initialization: Select an arbitrary point $\left(\boldsymbol{\theta}^{(0)}, s^{(0)}\right)$, and set $i=0$.

2. Gibbs sampler: Generate $s^{(i+1)}$ from the posterior $\pi\left(s \mid \boldsymbol{\theta}^{(i)}, \mathbf{g}\right)$ with Gamma distribution,

$$
s^{(i+1)} \sim \operatorname{Gamma}\left(a^{*}, b^{*}\right), \quad a^{*}=a+N, \quad b^{*}=b+d_{W}\left(\mathbf{f}\left(\boldsymbol{\theta}^{(i)}\right), \mathbf{g}\right) .
$$

3. Metropolis-Hastings sampler: Generate $\boldsymbol{\theta}^{(i+1)}$ as follows:

- Sample a candidate $\tilde{\boldsymbol{\theta}}$ from a proposal distribution $q\left(\boldsymbol{\theta}^{(i)}, \tilde{\boldsymbol{\theta}}\right)$.

- Compute the ratio

$$
\alpha\left(\boldsymbol{\theta}^{(i)}, \tilde{\boldsymbol{\theta}}\right)=\frac{\pi_{\exp }\left(\mathbf{g} \mid \tilde{\boldsymbol{\theta}}, s^{(i+1)}\right) \pi_{\text {prior }}(\tilde{\boldsymbol{\theta}}) q\left(\boldsymbol{\theta}^{(i)}, \tilde{\boldsymbol{\theta}}\right)}{\pi_{\exp }\left(\mathbf{g} \mid \boldsymbol{\theta}^{(i)}, s^{(i+1)}\right) \pi_{\text {prior }}\left(\boldsymbol{\theta}^{(i)}\right) q\left(\tilde{\boldsymbol{\theta}}, \boldsymbol{\theta}^{(i)}\right)},
$$

with the likelihood $\pi_{\exp }$ and the prior $\pi_{\text {prior }}$ given in (5) and (6), respectively.

- Set

$$
\boldsymbol{\theta}^{(i+1)}= \begin{cases}\tilde{\boldsymbol{\theta}} & \text { if } \operatorname{Unif}(0,1) \leq \alpha\left(\boldsymbol{\theta}^{(i)}, \tilde{\boldsymbol{\theta}}\right), \\ \boldsymbol{\theta}^{(i)} & \text { otherwise. }\end{cases}
$$

4. Iteration: Increment $i$ by 1 and go to step 2 . 


\section{Application to seismic inversion}

In this section, we apply the proposed Bayesian framework to the inverse uncertainty quantification of wave propagation problems. More specifically, we consider two problems: source inversion and material inversion. We present several numerical examples and demonstrate the applicability and performance of the proposed framework in comparison with the conventional Bayesian framework based on Gaussian likelihoods.

\subsection{A one-dimensional source inversion problem}

This example concerns a simple one-dimensional source inversion problem where an initial wave pulse propagates with a constant speed. The purpose of this example is to demonstrate the two major advantages of the Wasserstein distance over the standard least-squares norm separately, i.e. 1) handling complex noise structures, and 2) handling the problem with phase shifts and the cycle skipping issue. Correspondingly, we split the example into two parts: A) with a complicated noise structure and unknown amplitude, and B) with additive Gaussian noise and unknown phase and amplitude. Since the only parameter in part A is the amplitude, there will be no phase shift and no cycle skipping issue. Therefore, part $\mathrm{A}$ is designed to demonstrate the advantage of the Wasserstein distance only in handling complicated noise structures. In part B, on the other hand, noise is not an issue. With phase being an unknown, part $\mathrm{B}$ focuses on the performance of the two distances in handling the cycle skipping issue and the presence of multiple local minima.

Problem formulation. Consider the Cauchy problem for the 1D wave equation

$$
\begin{aligned}
& u_{t t}(t, x)-u_{x x}(t, x)=0, \quad t \in[0, T], \quad x \in \mathbb{R}, \\
& u(0, x)=h\left(x ; x_{0}, a\right), \quad u_{t}(0, x)=0 .
\end{aligned}
$$

The initial data

$$
h\left(x ; x_{0}, a\right)=a\left(e^{-100\left(x-x_{0}-0.5\right)^{2}}+e^{-100\left(x-x_{0}\right)^{2}}+e^{-100\left(x-x_{0}+0.5\right)^{2}}\right),
$$

acts as a source term generating an initial wave pulse, involving two parameters: the initial location $x_{0}$ and the amplitude $a$ of the wave pulse. The solution to this simple problem is given by d'Alembert's formula

$$
u\left(t, x ; x_{0}, a\right)=\frac{1}{2} h\left(x-t ; x_{0}, a\right)+\frac{1}{2} h\left(x+t ; x_{0}, a\right) .
$$

\subsubsection{A complicated noise structure and unknown amplitude}

Synthetic data. Let $x_{0}=0$ and $\theta=a$ be the only unknown parameter. Consider a uniform array of $N_{r}=7$ receivers located at

$$
x_{1}=-3, \quad x_{2}=-2, \quad x_{3}=-1, \quad x_{4}=0, \quad x_{5}=1, \quad x_{6}=2, \quad x_{7}=3 .
$$

Each receiver located at $x_{r}$, with $r=1, \ldots, N_{r}$, records a noisy discrete-time signal $g\left(t_{k}, x_{r}\right)$ over the time interval $[0, T]$ at $N$ discrete time levels $t_{k}=(k-1) \Delta t$, with $\Delta t=T /(N-1)$ 
and $k=1, \ldots, N$. Let $f\left(t_{k}, x_{r} ; \theta\right)$ denote the corresponding simulated signal for a given $\theta$, computed by (10) with $x_{0}=0$. We select a fixed parameter

$$
\theta^{*}=5
$$

and generate synthetic data as follows. We first compute $f\left(t_{k}, x_{r} ; \theta^{*}\right)$ by 10 and then generate $g\left(t_{k}, x_{r}\right)$ by polluting $f\left(t_{k}, x_{r} ; \theta^{*}\right)$ with a noise of the form (1): we multiply $f$ by i.i.d. Gamma noise and then add an i.i.d uniform noise to the product, that is,

$$
g\left(t_{k}, x_{r}\right)=\varepsilon_{k r}^{(1)} f\left(t_{k}, x_{r} ; \theta^{*}\right)+\varepsilon_{k r}^{(2)}, \quad \varepsilon_{k r}^{(1)} \sim \operatorname{Gamma}(60,60), \quad \varepsilon_{k r}^{(2)} \sim \operatorname{Unif}(-0.25,0.25) .
$$

Let $\mathbf{g}_{r} \in \mathbb{R}^{N}$ denote the discrete-time signal recorded at $x_{r}, r=1, \ldots, N_{r}$. We have a set of $N_{r}=7$ such signals, shown in Figure 4 with $T=5$ and $N=101$. Similarly, we let $\mathbf{f}_{r}(\theta) \in \mathbb{R}^{N}$ be the $r$-th simulated signal, consisting of $f\left(t_{k}, x_{r} ; \theta\right)$ at all $N$ discrete time levels $\left\{t_{k}\right\}_{k=1}^{N}$.
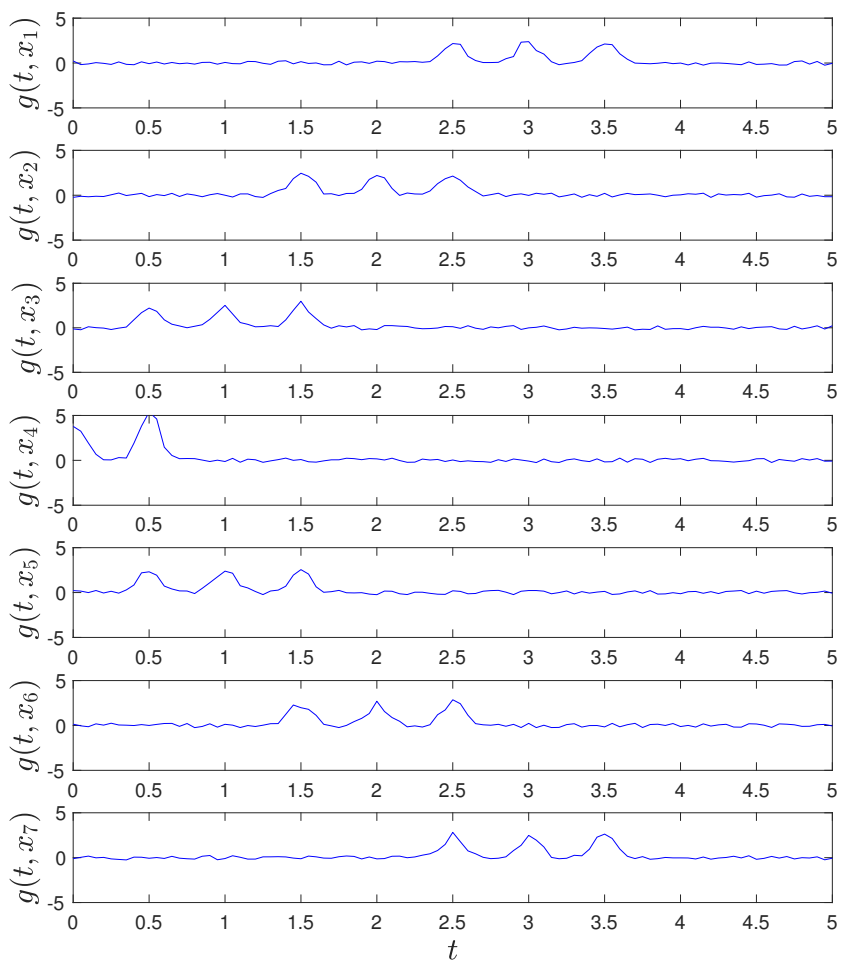

Figure 2: Observed signals with complex noise structures, recorded at seven receivers.

Computations. The goal is to employ Bayesian inversion and compute the conditional posterior of the amplitude parameter $\theta$. We follow Algorithm 1 with the following choices:

- Likelihood: $\pi_{\exp }(\mathbf{g} \mid \theta)=s^{N} \exp \left(-s \sum_{r=1}^{N_{r}} d_{W}\left(\mathbf{f}_{r}(\theta), \mathbf{g}_{r}\right)\right)$.

- Priors: $\theta \sim \operatorname{Unif}(2,8), \quad s \sim \operatorname{Gamma}(1,0.1)$.

- Proposal: A Gaussian random walk (7) with variance $\Sigma=0.005$. 
- Initial point: $\theta^{(0)}=3, \quad s^{(0)}=70$.

We also consider the standard normal likelihood based on the Euclidean distance:

$$
\pi_{\text {norm }}(\mathbf{g} \mid \theta)=\frac{s^{N / 2}}{(2 \pi)^{N / 2}} \exp \left(-\frac{s}{2} \sum_{r=1}^{N_{r}} d_{2}\left(\mathbf{f}_{r}(\theta), \mathbf{g}_{r}\right)\right), \quad s=\sigma^{-2},
$$

where $d_{2}\left(\mathbf{f}_{r}(\theta), \mathbf{g}_{r}\right)=\sum_{k=1}^{N}\left|g\left(t_{k}, x_{r}\right)-f\left(t_{k}, x_{r} ; \theta\right)\right|^{2}$. We note that in this case $s=\sigma^{-2}$ will be the precision parameter.

We run the algorithm with $M_{0}=30000$ iterations and remove the first $M_{b}=10000$ samples, known as the burn-in period. We also use a thinning period of $M_{t}=4$, that is, we keep every 4 th samples and discard the rest. This would give a total of $M=5000$ Markov chain samples. Figure 5 shows the posterior histograms and the trace plots of the Markov chain samples generated by the algorithm with both (a) the proposed likelihood and (b) the Gaussian likelihood. We observe that while both posteriors are centered around the true noise-free parameter, the one obtained by the proposed exponential likelihood function is much more concentrated compared to the one obtained by the Gaussian likelihood function. This clearly demonstrate the power of the proposed likelihood in capturing more information in the available data compared to standard Gaussian likelihood.
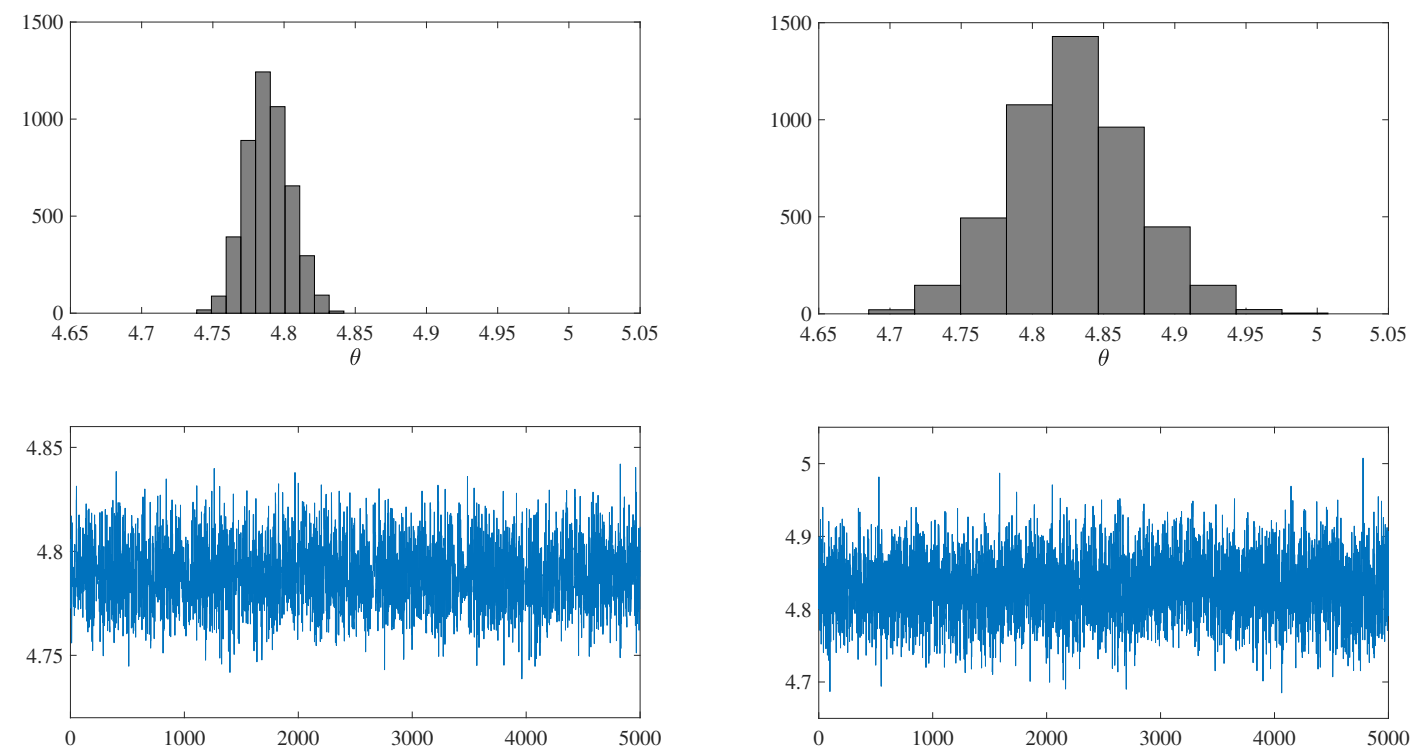

(a) Wasserstein-driven exponential likelihood.

(b) $L_{2}$-driven Gaussian likelihood.

Figure 3: Posterior histograms and the trace plots of the Markov chain samples in two cases: (a) with the proposed Wasserstein-driven exponential likelihood, and (b) with the standard Gaussian likelihood. Clearly, the posterior obtained by the Gaussian likelihood function is much more concentrated compared to the one obtained by the Gaussian likelihood function. 


\subsubsection{An additive Gaussian noise and unknown phase and amplitude}

Synthetic data. Let $\boldsymbol{\theta}=\left(x_{0}, a\right)$ be the unknown parameter vector. Consider a uniform array of $N_{r}=7$ receivers located at

$$
x_{1}=-3, \quad x_{2}=-2, \quad x_{3}=-1, \quad x_{4}=0, \quad x_{5}=1, \quad x_{6}=2, \quad x_{7}=3 .
$$

Each receiver located at $x_{r}$, with $r=1, \ldots, N_{r}$, records a noisy discrete-time signal $g\left(t_{k}, x_{r}\right)$ over the time interval $[0, T]$ at $N$ discrete time levels $t_{k}=(k-1) \Delta t$, with $\Delta t=T /(N-1)$ and $k=1, \ldots, N$. Let $f\left(t_{k}, x_{r} ; \boldsymbol{\theta}\right)$ denote the corresponding simulated signal for a given $\boldsymbol{\theta}$, computed by 10$)$. We select a fixed parameter vector

$$
\boldsymbol{\theta}^{*}=(0,5)
$$

and generate synthetic data as follows. We first compute $f\left(t_{k}, x_{r} ; \boldsymbol{\theta}^{*}\right)$ by (10) and then generate $g\left(t_{k}, x_{r}\right)$ by polluting $f\left(t_{k}, x_{r} ; \boldsymbol{\theta}^{*}\right)$ with an additive Gaussian noise,

$$
g\left(t_{k}, x_{r}\right)=f\left(t_{k}, x_{r} ; \boldsymbol{\theta}^{*}\right)+\varepsilon_{k r}, \quad \varepsilon_{k r} \sim \operatorname{Normal}(0,0.1) .
$$

Let $\mathbf{g}_{r} \in \mathbb{R}^{N}$ denote the discrete-time signal recorded at $x_{r}, r=1, \ldots, N_{r}$. We have a set of $N_{r}=7$ such signals, shown in Figure 4 with $T=5$ and $N=101$. Similarly, we let $\mathbf{f}_{r}(\boldsymbol{\theta}) \in \mathbb{R}^{N}$ be the $r$-th simulated signal, consisting of $f\left(t_{k}, x_{r} ; \boldsymbol{\theta}\right)$ at all $N$ discrete time levels $\left\{t_{k}\right\}_{k=1}^{N}$.

Computations. The goal is to employ Bayesian inversion and compute the conditional posterior of the model parameters $\boldsymbol{\theta}$. We follow Algorithm 1 with the following choices:

- Likelihood: $\pi_{\exp }(\mathbf{g} \mid \boldsymbol{\theta})=s^{N} \exp \left(-s \sum_{r=1}^{N_{r}} d_{W}\left(\mathbf{f}_{r}(\boldsymbol{\theta}), \mathbf{g}_{r}\right)\right)$.

- Priors: $\theta_{1} \sim \operatorname{Unif}(-3,3), \quad \theta_{2} \sim \operatorname{Unif}(2,8), \quad s \sim \operatorname{Gamma}(1,0.1)$.

- Proposal: A Gaussian random walk (7) with covariance $\Sigma=\operatorname{diag}(0.005,0.005)$.

- Initial point: $\boldsymbol{\theta}^{(0)}=(0.6,3), \quad s^{(0)}=70$.

We also consider the standard normal likelihood based on the Euclidean distance:

$$
\pi_{\text {norm }}(\mathbf{g} \mid \boldsymbol{\theta})=\frac{s^{N / 2}}{(2 \pi)^{N / 2}} \exp \left(-\frac{s}{2} \sum_{r=1}^{N_{r}} d_{2}\left(\mathbf{f}_{r}(\boldsymbol{\theta}), \mathbf{g}_{r}\right)\right), \quad s=\sigma^{-2},
$$

where $d_{2}\left(\mathbf{f}_{r}(\boldsymbol{\theta}), \mathbf{g}_{r}\right)=\sum_{k=1}^{N}\left|g\left(t_{k}, x_{r}\right)-f\left(t_{k}, x_{r} ; \boldsymbol{\theta}\right)\right|^{2}$. We note that in this case $s=\sigma^{-2}$ will be the precision parameter.

We run the algorithm with $M_{0}=25000$ iterations and remove the first $M_{b}=5000$ samples, known as the burn-in period. We also use a thinning period of $M_{t}=4$, that is, we keep every 4 th samples and discard the rest. This would give a total of $M=5000$ Markov chain samples. Figure 5 shows the posterior histograms and the trace plots of the Markov chain samples generated by the algorithm with both (a) the proposed likelihood and (b) the Gaussian likelihood. We observe that the samples obtained by the standard Gaussian likelihood converge to wrong distributions, while the Markov chain samples obtained by the 

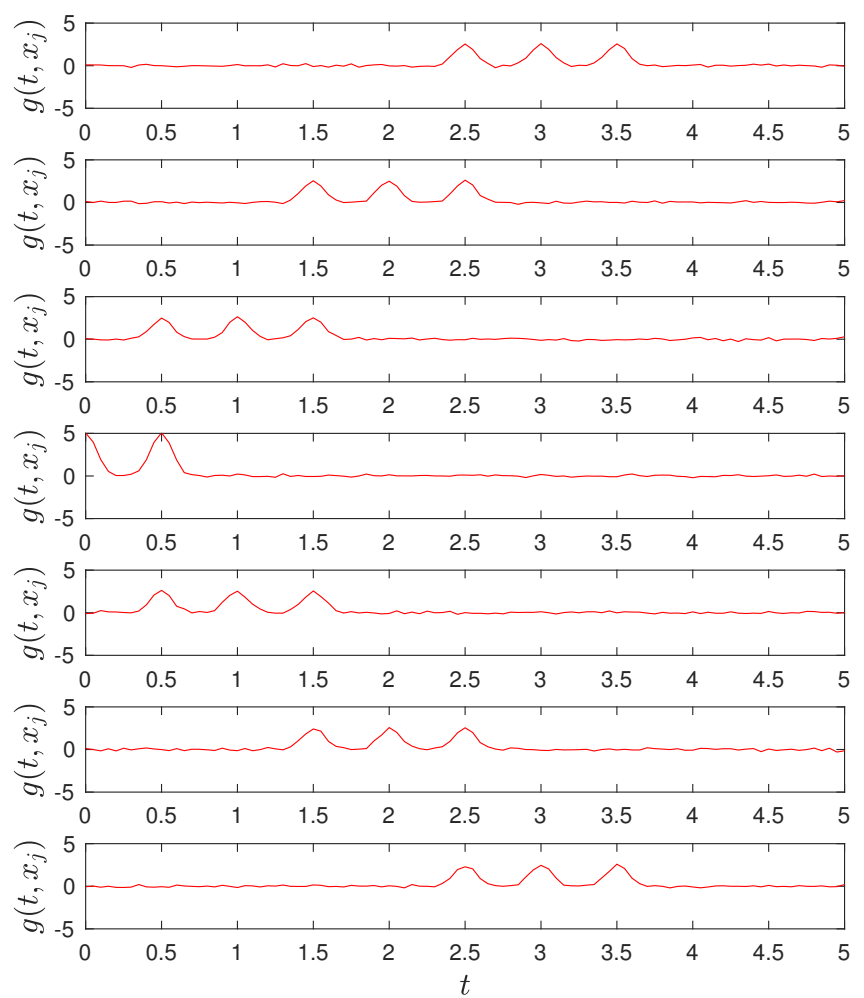

Figure 4: Observed signals with additive Gaussian noise, recorded at seven receivers.

proposed exponential likelihood function are centered around the true, noise-free parameters.

The wrong convergence of the standard Bayesian approach, as shown in Figure 5, is due to the existence of multiple local maxima in the Gaussian likelihood function. Consequently, the Markov chain samples may get trapped in a local maximum and hence miss the global maximum. To illustrate this, in Figure 6 we plot the $\log$-likelihood functions $\log L_{\exp }\left(\theta_{1}, 5\right)$ and $\log L_{\text {norm }}\left(\theta_{1}, 5\right)$ versus $\theta_{1} \in[-3,3]$. We observe that the Gaussian likelihood has multiple local maxima, while the proposed exponential likelihood has a more-convex shape, making it easy for MCMC to infer its global maximum.

\subsection{A two-dimensional seismic source inversion problem}

This example concerns the inference of a seismic source in a layered isotropic elastic material in two dimensions. The source models a simplified earthquake, represented by four unknown parameters.

Problem formulation. We consider the initial-boundary value problem (IBVP) for the 
elastodynamic wave equation,

$$
\begin{array}{ll}
\nu(\mathbf{x}) \mathbf{u}_{t t}(t, \mathbf{x})-\nabla \cdot \boldsymbol{\sigma}(\mathbf{u}(t, \mathbf{x}))=\mathbf{h}(t, \mathbf{x} ; \boldsymbol{\theta}) & \text { in }[0, T] \times D \\
\mathbf{u}(0, \mathbf{x})=\mathbf{0}, \quad \mathbf{u}_{t}(0, \mathbf{x})=\mathbf{0} & \text { on }\{t=0\} \times D \\
\boldsymbol{\sigma}(\mathbf{u}(t, \mathbf{x})) \cdot \hat{\mathbf{n}}=\mathbf{0} & \text { on }[0, T] \times \partial D_{0} \\
\mathbf{u}_{t}(t, \mathbf{x})=B(\mathbf{x}) \boldsymbol{\sigma}(\mathbf{u}(t, \mathbf{x})) \cdot \hat{\mathbf{n}} & \text { on }[0, T] \times \partial D_{1}
\end{array}
$$

The solution $\mathbf{u}=\left(u_{1}, u_{2}\right)^{\top}$ represents the displacement field, $t \in[0, T]$ is the time, $\mathbf{x}=$ $\left(x_{1}, x_{2}\right) \in D$ is the location, and $\boldsymbol{\sigma}$ is the stress tensor given by

$$
\boldsymbol{\sigma}(\mathbf{u})=\lambda(\mathbf{x}) \nabla \cdot \mathbf{u} I+\mu(\mathbf{x})\left(\nabla \mathbf{u}+(\nabla \mathbf{u})^{\top}\right)
$$

where $I$ is the identity matrix. The computational domain is a box $D=[-10000,10000] \times$ $[-15000,0]$, consisting of two layers: the top layer $D_{I}$ extends over $-1000 \leq x_{2} \leq 0$, and the bottom layer $D_{I I}$ is given by $x_{2} \leq-1000$. The material properties are characterized by the density $\nu$ and the Lamé parameters, $\lambda$ and $\mu$. The system (11) admits longitudinal (or pressure) and transverse (or shear) waves, which, in the case of constant density, propagate at the wave speeds

$$
c_{p}=\sqrt{(2 \mu+\lambda) / \nu}, \quad c_{s}=\sqrt{\mu / \nu}
$$

respectively. The material density and velocities are assumed to be known, given by

$$
\nu(\mathbf{x})=\left\{\begin{array}{ll}
2600 & \mathbf{x} \in D_{I} \\
2700 & \mathbf{x} \in D_{I I}
\end{array} \quad, \quad c_{p}(\mathbf{x})=\left\{\begin{array}{ll}
4000 & \mathbf{x} \in D_{I} \\
6000 & \mathbf{x} \in D_{I I}
\end{array}, \quad c_{s}(\mathbf{x})=\left\{\begin{array}{ll}
2000 & \mathbf{x} \in D_{I} \\
3464 & \mathbf{x} \in D_{I I}
\end{array} .\right.\right.\right.
$$

We impose a homogeneous Neumann (stress-free) boundary condition (11c) on the free surface $\partial D_{0}=\left\{\mathbf{x} \mid x_{2}=0\right\}$, and the first-order Clayton-Engquist non-reflecting boundary conditions [8] on the other three boundaries $\partial D_{1}=\partial D \backslash \partial D_{0}$. Here, $\hat{\mathbf{n}}$ is the outward unit normal to the boundary, and $B$ is a given matrix related to the non-reflecting boundary condition.

The function $\mathbf{h}$ represents the seismic source. We consider the case of a point moment tensor source that models a simplified earthquake,

$$
\mathbf{h}(t, \mathbf{x} ; \boldsymbol{\theta})=S(t) M \nabla \delta\left(\mathbf{x}-\mathbf{x}_{s}\right)
$$

located at $\mathbf{x}_{s}=\left(0, z_{s}\right)$, where $\nabla \delta$ is the gradient of the Dirac distribution. The source time function $S(t)$ and the moment tensor are assumed to be

$$
S(t)=\frac{\omega_{s}}{\sqrt{2 \pi}} e^{-\omega_{s}^{2}\left(t-t_{s}\right)^{2} / 2}, \quad M=m_{s}\left(\begin{array}{cc}
10^{10} & 0.8 \times 10^{10} \\
0.8 \times 10^{10} & 10^{10}
\end{array}\right) .
$$

parametrized by the frequency $\omega_{s}$, the center time $t_{s}$, and a constant $m_{s}$. Hence, the source parameter vector $\boldsymbol{\theta}$ consists of four parameters,

$$
\boldsymbol{\theta}=\left(z_{s}, t_{s}, \omega_{s}, m_{s}\right)
$$

Synthetic data. We place a uniform array of $N_{r}=90$ receivers located at

$$
\mathbf{x}_{r}=\left(x_{1, r}, x_{2, r}\right)=(-8900+200(r-1), 0), \quad r=1,2, \ldots, N_{r}
$$


Figure 7 shows the computational domain and the configuration of the problem. Each receiver located at $\mathbf{x}_{r}$, with $r=1, \ldots, N_{r}$, records a noisy vector-valued discrete-time signal $\bar{g}\left(t_{k}, \mathbf{x}_{r}\right)=$ $\left(g_{1}\left(t_{k}, \mathbf{x}_{r}\right), g_{2}\left(t_{k}, \mathbf{x}_{r}\right)\right)$ over the time interval $[0, T]$ at $N$ discrete time levels $t_{k}=(k-1) \Delta t$, with $\Delta t=T /(N-1)$ and $k=1, \ldots, N$. Moreover, let $\bar{f}\left(t_{k}, \mathbf{x}_{r} ; \boldsymbol{\theta}\right)=\left(f_{1}\left(t_{k}, \mathbf{x}_{r} ; \boldsymbol{\theta}\right), f_{2}\left(t_{k}, \mathbf{x}_{r} ; \boldsymbol{\theta}\right)\right)$ denote the corresponding simulated signal for a given $\boldsymbol{\theta}$ and computed by numerically solving (11). We employ a second-order accurate finite difference scheme, proposed in [27], with grid size $\Delta x_{1}=\Delta x_{2}=100$ and time step $\Delta t=0.0125$. We set the final time $T=8$ with $N=641$ time steps. We also employ the technique proposed in [29] for regularized approximations of the Dirac distribution and its gradient to achieve point-wise convergence of the solution away from the singular source. We refer to [20] for details of the employed forward solver. We then choose a fixed parameter vector

$$
\boldsymbol{\theta}^{*}=\left(-2000,1,4,10^{4}\right)
$$

and generate synthetic data as follows. We first numerically compute $\bar{f}\left(t_{k}, \mathbf{x}_{r} ; \boldsymbol{\theta}^{*}\right)$ and then generate $\bar{g}\left(t_{k}, \mathbf{x}_{r}\right)$ by polluting $\bar{f}\left(t_{k}, \mathbf{x}_{r} ; \boldsymbol{\theta}^{*}\right)$ with a noise of the form (1):

$g_{1,2}\left(t_{k}, \mathbf{x}_{r}\right)=\varepsilon_{k r}^{(1)} f_{1,2}\left(t_{k}, \mathbf{x}_{r} ; \boldsymbol{\theta}^{*}\right)+\varepsilon_{k r}^{(2)}, \quad \varepsilon_{k r}^{(1)} \sim \operatorname{Gamma}(500,500), \quad \varepsilon_{k r}^{(2)} \sim \operatorname{Unif}\left(-\frac{1}{80}, \frac{1}{80}\right)$.

Let $\mathbf{g}_{r} \in \mathbb{R}^{N}$ denote the discrete-time signal recorded at the receiver location $\mathbf{x}_{r}$, with $r=$ $1, \ldots, N_{r}$. We note that since there are two (a horizontal and a vertical) components of the solution, we have a set of $2 N_{r}=180$ such signals. Similarly, we let $\mathbf{f}_{r}(\boldsymbol{\theta}) \in \mathbb{R}^{N}$ be the $r$-th simulated signal, consisting of $f_{1}\left(t_{k}, \mathbf{x}_{r} ; \boldsymbol{\theta}\right)$ or $f_{2}\left(t_{k}, \mathbf{x}_{r} ; \boldsymbol{\theta}\right)$ at all $N$ discrete time levels $\left\{t_{k}\right\}_{k=1}^{N}$.

Computations. The goal is to employ Bayesian inversion and compute the conditional posterior of the model parameters $\boldsymbol{\theta}$. We follow Algorithm 1 with the following choices:

- Likelihood: $\pi_{\exp }(\mathbf{g} \mid \boldsymbol{\theta})=s^{N} \exp \left(-s \sum_{r=1}^{2 N_{r}} d_{W}\left(\mathbf{f}_{r}(\boldsymbol{\theta}), \mathbf{g}_{r}\right)\right)$.

- Priors: $\quad \theta_{1} \sim \operatorname{Unif}(-2200,-1800), \quad \theta_{2} \sim \operatorname{Unif}(0.5,1.5), \quad \theta_{3} \sim \operatorname{Unif}(2,6)$,

$$
\theta_{4} \sim \operatorname{Unif}(9600,10400), \quad s \sim \operatorname{Gamma}(1,0.1) .
$$

- Proposal: A Gaussian random walk (7) with covariance $\Sigma$, which is initially chosen to be diagonal and then updated based on the first 1000 MCMC samples.

- Initial point: $\boldsymbol{\theta}^{(0)}=(-2100,0.7,3,9800), \quad s^{(0)}=2000$.

We run the algorithm with $M_{0}=10000$ iterations and remove the first $M_{b}=2000$ samples. We also use a thinning period of $M_{t}=4$. This would give a total of $M=2000$ Markov chain samples. Figure 8 shows the posterior histograms and the trace plots of the Markov chain samples generated by the algorithm.

It is to be noted that similar to the first numerical example, we observed wrong convergence of the samples using the standard Gaussian likelihood function. 


\subsection{A two-dimensional material inversion problem}

This example concerns the inference of wave speed in a two dimensional spatial domain, consisting of ten non-overlapping sub-domains, each with a different unknown wave speed.

Problem formulation. Consider the initial-boundary value problem for the acoustic wave equation in a two-dimensional spatial domain $D=[-1,1] \times[-2,0]$ :

$$
\begin{array}{ll}
u_{t t}(t, \mathbf{x})-\nabla_{\mathbf{x}} \cdot\left(a^{2}(\mathbf{x} ; \boldsymbol{\theta}) \nabla_{\mathbf{x}} u(t, \mathbf{x})=F_{s}(t, \mathbf{x}),\right. & t \in[0, T], \quad \mathbf{x} \in D \\
u(t, \mathbf{x})=0, \quad u_{t}(t, \mathbf{x})=0, & t=0, \quad \mathbf{x} \in D \\
u(t, \mathbf{x})=0, & t \in[0, T], \quad \mathbf{x} \in \partial D
\end{array}
$$

The source $F_{s}$ is a Ricker wavelet applied on a square $D_{s}$ of size $0.08 \times 0.08$ centered at a given point $\left(x_{1, s}, x_{2, s}\right)$ :

$$
F_{s}(t, \mathbf{x})= \begin{cases}10^{3}\left(1-20(t-0.1)^{2}\right) \exp \left(-10(t-0.1)^{2}\right), & \mathbf{x} \in D_{s} \\ 0, & \mathbf{x} \notin D_{s}\end{cases}
$$

The wave speed $a$ is assumed to be described by ten (unknown) parameters $\boldsymbol{\theta}=\left(\theta_{1}, \ldots, \theta_{10}\right)$. The computational domain $D$ is split into ten non-overlapping sub-domains of size $0.4 \times 1$, each with a constant velocity $a=\theta_{i}$, with $i=1, \ldots, 10$; see Figure 9 .

Synthetic data. The acquisition geometry consists of $N_{s}=5$ sources $F_{s}$ evenly centered at depth $x_{2}=-0.2$ along the horizontal line $x_{1} \in[-0.8,0.8]$, and $N_{r}=201$ evenly spaced receivers at the surface $\left(x_{2}=0\right)$ with a spacing of 0.02 and the left $\left(x_{1}=-1\right)$ and right $\left(x_{1}=1\right)$ boundaries with a spacing of 0.04 . Figure 9 shows the computational domain and the configuration of the problem.

Each receiver located at $\mathbf{x}_{r}$, with $r=1, \ldots, N_{r}$, records a (noisy) discrete-time signal $g_{s}\left(t_{k}, \mathbf{x}_{r}\right)$, due to a single source $F_{s}$, with $s=1, \ldots, N_{s}$, and over the time interval $[0, T]$ at $N$ discrete time levels $t_{k}=(k-1) \Delta t$, with $\Delta t=T /(N-1)$ and $k=1, \ldots, N$. Moreover, let $f_{s}\left(t_{k}, \mathbf{x}_{r} ; \boldsymbol{\theta}\right)$ denote the corresponding simulated signal for a given $\boldsymbol{\theta}$, obtained by employing a finite difference scheme based on the second-order central difference discretization of (14); see [26] for details of the deterministic solver. We use a uniform grid with spatial grid-lengths $\Delta x_{1}=\Delta x_{2}=0.02$ and time step $\Delta t=0.005$. We set the final time $T=4$ with $N=801$ time steps. We choose a fixed parameter vector

$$
\boldsymbol{\theta}^{*}=(3,2,3.5,2.5,4,3,4.5,3.5,5,4)
$$

and generate synthetic data by first computing $f_{s}\left(t_{k}, \mathbf{x}_{r} ; \boldsymbol{\theta}^{*}\right)$ and then polluting it with a noise of the form (1):

$g_{s}\left(t_{k}, \mathbf{x}_{r}\right)=\varepsilon_{s k r}^{(1)} f_{s}\left(t_{k}, \mathbf{x}_{r} ; \boldsymbol{\theta}^{*}\right)+\varepsilon_{s k r}^{(2)}, \quad \varepsilon_{s k r}^{(1)} \sim \operatorname{Gamma}(1000,1000), \quad \varepsilon_{s k r}^{(2)} \sim \operatorname{Unif}(-0.05,0.05)$.

Let $\mathbf{g}_{s r} \in \mathbb{R}^{N}$ and $\mathbf{f}_{s r}(\boldsymbol{\theta}) \in \mathbb{R}^{N}$ denote the vectors of recorded and simulated discrete-time signals due to a single source $F_{s}, s=1, \ldots, N_{s}$, at the receiver location $\mathbf{x}_{r}, r=1, \ldots, N_{r}$. Note that since there are $N_{s}=5$ single sources, we have in total $N_{s} N_{r}=1005$ such signals.

Computations. The goal is to employ Bayesian inversion and compute the conditional posterior of the model parameter vector $\boldsymbol{\theta}=\left(\theta_{1}, \ldots, \theta_{10}\right)$. We follow Algorithm 1 with the following choices: 
- Likelihood: $\pi_{\exp }(\mathbf{g} \mid \boldsymbol{\theta})=s^{N} \exp \left(-s \sum_{s=1}^{N_{s}} \sum_{r=1}^{N_{r}} d_{W}\left(\mathbf{f}_{s r}(\boldsymbol{\theta}), \mathbf{g}_{s r}\right)\right)$.

- Priors: $\theta_{j} \sim \operatorname{Unif}(1,6), j=1, \ldots, 10, \quad s \sim \operatorname{Gamma}(1,0.1)$.

- Proposal: A Gaussian random walk (7) with covariance $\Sigma$, which is initially chosen to be diagonal and then updated based on the first 1000 MCMC samples.

- Initial point: $\theta_{j}^{(0)}=3.8, \quad j=1, \ldots, 10, \quad s^{(0)}=5000$.

We run the algorithm with $M_{0}=80000$ iterations and remove the first $M_{b}=65000$ samples. We also use a thinning period of $M_{t}=3$. This would give a total of $M=5000$ Markov chain samples. Figure 10 shows the posterior histograms of the Markov chain samples generated by the proposed algorithm.

Figure 11 shows a map of the original noise-free parameters (a), the mean map (b) and the standard deviation map (c) obtained by the generated MCMC samples of the parameter posteriors.

\section{Conclusion}

We have presented a robust Bayesian framework based on a new exponential likelihood function driven by the quadratic Wasserstein metric. We demonstrated that this framework is able to treat complicated noise structures and presented several numerical examples subject to combined additive and multiplicative noise. As in the deterministic setting we observe that the convexity properties of the Wasserstein metric translates into better convergence, here towards the correct posteriors.

It is to be noted that in this work we exclusively used a linear "shift and rescale" approach to convert signals into positive probabilities. Although this procedure was shown to produce good results, there may be other alternatives, e.g. the combination of the exponential and linear procedure as discussed in the end of Section 3, that could prove to be more suitable for certain problems. A detailed exploration of the impact of normalization on the proposed method is a subject of our future work.

As the framework presented here is particularly suitable for wave propagation problems we also plan to apply it to geoacoustic inversion of water and seafloor parameters in shallow water range dependent environments. There the material properties vary smoothly and consequently the forward problems can be efficiently simulated with our arbitrary order wave solvers [1].

Other future directions include the application of Wasserstein distance to other Bayesian models, such as variational inference (see e.g. [5]), that suggest alternative strategies to MCMC sampling and hence tend to be faster to scale to large data.

Acknowledgements. The authors are indebted to Dr. Gabriel Huerta at Sandia National Laboratories for his guidance and stimulating discussions. We also thank the reviewers for their constructive comments. 


\section{References}

[1] D. Appelö, T. Hagstrom, and A. Vargas. Hermite methods for the scalar wave equation. SIAM Journal on Scientific Computing, 40(6):A3902-A3927, 2018.

[2] M. Ballesio, J. Beck, A. Pandey, L. Parisi, E. von Schwerin, and R. Tempone. Multilevel Monte Carlo Acceleration of Seismic Wave Propagation under Uncertainty. arXiv eprints, page arXiv:1810.01710, October 2018.

[3] T. Bayes, R. Price, and J. Canton. An essay towards solving a problem in the doctrine of chances. By the late Rev. Mr. Bayes, F. R. S. communicated by Mr. Price, in a letter to John Canton, A. M. F. R. Philosophical Transactions of the Royal Society of London, pages 370-418, 1763.

[4] J.-D. Benamou, B. D. Froese, and A. M. Oberman. Numerical solution of the Optimal Transportation problem using the Monge-Ampère equation. Journal of Computational Physics, 260:107-126, 2014.

[5] D. M. Blei, A. Kucukelbir, and J. D. McAuliffe. Variational inference: A review for statisticians. Journal of the American Statistical Association, 112:859-877, 2017.

[6] J. Chen, Y. Chen, H. Wu, and D. Yang. The quadratic Wasserstein metric for earthquake location. J. Comput. Physics, 373:188-209, 2018.

[7] S. Chib and E. Greenberg. Understanding the Metropolis-Hastings algorithm. The American Statistician, 49:327-335, 1995.

[8] R. Clayton and B. Engquist. Absorbing boundary conditions for acoustic and elastic wave equations. Bull. Seismol. Soc. Amer., 67:1529-1540, 1977.

[9] B. Engquist, B.D. Froese Brittany, and Y. Yang. Optimal transport for seismic full waveform inversion. Communications in Mathematical Sciences, 14(8):2309-2330, 2016.

[10] B. Engquist and Y. Yang. Seismic Imaging and Optimal Transport. To appear in Communications in Information and Systems, see also arXiv e-prints, page arXiv:1808.04801, August 2018.

[11] Björn Engquist and Brittany D Froese. Application of the wasserstein metric to seismic signals. Communications in Mathematical Sciences, 12(5):979-988, 2014.

[12] P. Mohajerin Esfahani and D. Kuhn. Data-driven distributionally robust optimization using the Wasserstein metric: performance guarantees and tractable reformulations. Mathematical Programming, 2017.

[13] D. Gamerman and H. F. Lopes. Markov Chain Monte Carlo: Stochastic Simulation for Bayesian Inference. Chapman and Hall/CRC, 2006.

[14] A. Gelman, J. B. Carlin, H. S. Stern, and D. B. Rubin. Bayesian data analysis. Chapman and Hall/CRC, 2004. 
[15] J. Goodman. Speckle Phenomena in Optics: Theory and Applications. Roberts and Company Publishers, 2007.

[16] W. K. Hastings. Monte Carlo sampling methods using Markov chains and their applications. Biometrika, 57:97-109, 1970.

[17] N. Hedjazian, T. Bodin, and L. Métivier. An optimal transport approach to linearized inversion of receiver functions. Geophysical Journal International, 216(1):130-147, 2019.

[18] J. Kaipo and E. Somersalo. Statistical and Computational Inverse Problems. Springer, New York, 2005.

[19] S. Kolouri, Y. Zou, and G. K. Rohde. Sliced Wasserstein kernels for probability distributions. In 2016 IEEE Conference on Computer Vision and Pattern Recognition (CVPR), pages 5258-5267, June 2016.

[20] Q. Long, M. Motamed, and R. Tempone. Fast Bayesian optimal experimental design for seismic source inversion. Computer Methods in Applied Mechanics and Engineering, 291:123-145, 2015.

[21] L Métivier, A Allain, R Brossier, Q Mérigot, E Oudet, and J Virieux. A graph-space approach to optimal transport for full waveform inversion. In SEG Technical Program Expanded Abstracts 2018, pages 1158-1162. Society of Exploration Geophysicists, 2018.

[22] L. Métivier, A. Allain, R. Brossier, Q. Mérigot, E. Oudet, and J. Virieux. Optimal transport for mitigating cycle skipping in full-waveform inversion: A graph-space transform approach. GEOPHYSICS, 83(5):R515-R540, 2018.

[23] L. Métivier, R. Brossier, Q. Merigot, E. Oudet, and J. Virieux. Measuring the misfit between seismograms using an optimal transport distance: application to full waveform inversion. Geophysical Journal International, 205(1):345-377, 2016.

[24] L. Métivier, R. Brossier, Q. Merigot, E. Oudet, and J. Virieux. An optimal transport approach for seismic tomography: Application to $3 \mathrm{~d}$ full waveform inversion. Inverse Problems, 32(11):115008, 2016.

[25] N. Metropolis, A. W. Rosenbluth, M. N. Rosenbluth, A. H. Teller, and E. Teller. Equation of state calculations by fast computing machines. The Journal of Chemical Physics, 21:1087-1092, 1953.

[26] M. Motamed, F. Nobile, and R. Tempone. A stochastic collocation method for the second order wave equation with a discontinuous random speed. Numer. Math., 123:493-536, 2013.

[27] S. Nilsson, N. A. Petersson, B. Sjögren, and H.-O. Kreiss. Stable difference approximations for the elastic wave equation in second order formulation. SIAM J. Numer. Anal., 45:1902-1936, 2007. 
[28] N. Papadakis. Optimal transport for image processing. Habilitation, Signal and Image Processing, Université de Bordeaux, 2015. HAL Id: tel-01246096.

[29] B. Sjögreen and N. A. Petersson. Source estimation by full wave form inversion. Journal of Scientific Computing, 59:247-276, 2014.

[30] A. M. Stuart. Inverse problems: A Bayesian perspective. Acta Numerica, 19:451-559, 2010.

[31] C. Villani. Topics in Optimal Transportation, volume 58 of Graduate Studies in Mathematics. American Mathematical Society, Providence, Rhode Island, 2003.

[32] C. Villani. Optimal Transport: Old and New, volume 338 of Comprehensive Studies in Mathematics. Springer-Verlag, Berlin, 2009.

[33] Y. Yang, B. Engquist, J. Sun, and B. F. Hamfeldt. Application of optimal transport and the quadratic Wasserstein metric to full-waveform inversion. Geophysics, 83(1):R43R62, 2018. 

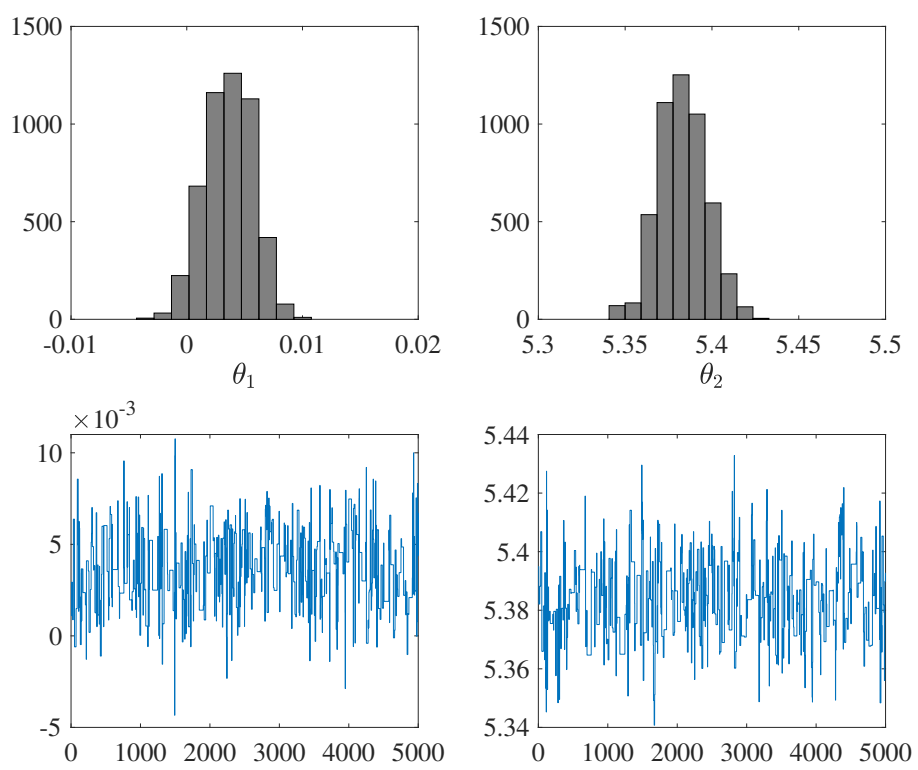

(a) Wasserstein-driven exponential likelihood.
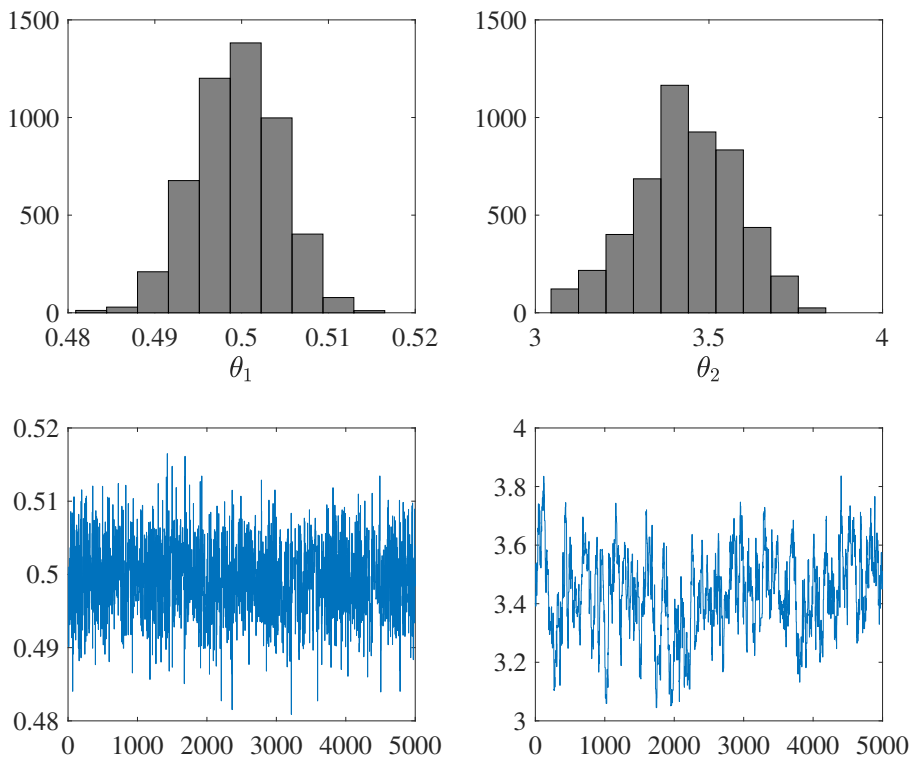

(b) $L_{2}$-driven Gaussian likelihood.

Figure 5: Posterior histograms and the trace plots of the Markov chain samples in two cases: (a) with the proposed Wasserstein-driven exponential likelihood, and (b) with the standard Gaussian likelihood. Clearly, the samples obtained by the Gaussian likelihood function do not converge to the correct distribution, while the samples obtained by the proposed likelihood are accumulated close to the true, noise-free parameters. 


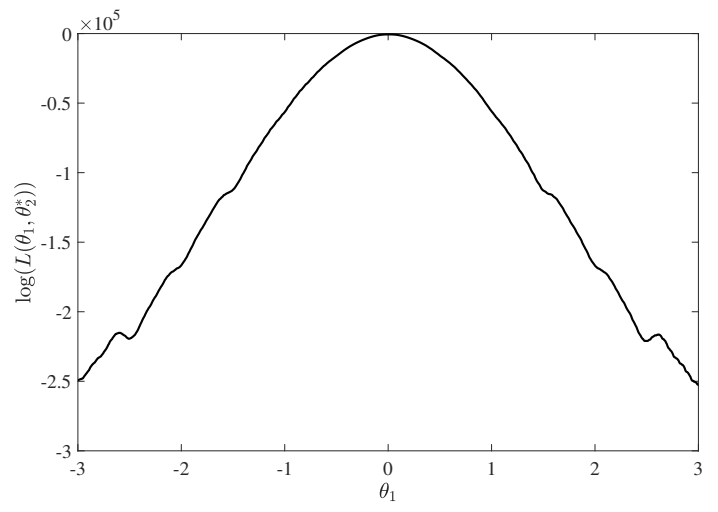

(a) Wasserstein-driven exponential likelihood.

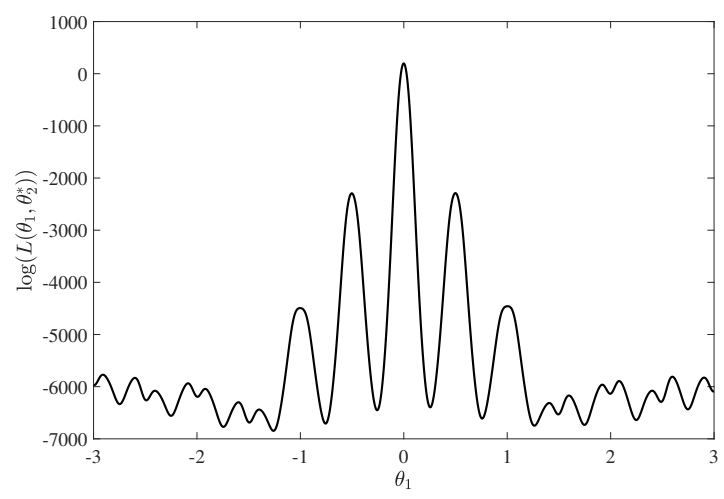

(b) $L_{2}$-driven Gaussian likelihood.

Figure 6: The $\log$-likelihood functions $\log L_{\text {exp }}\left(\theta_{1}, 5\right)$ and $\log L_{n o r m}\left(\theta_{1}, 5\right)$ versus $\theta_{1} \in[-3,3]$.

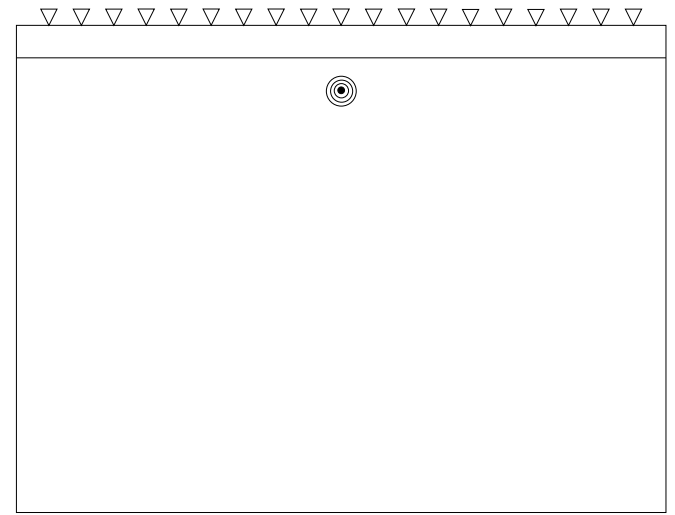

Figure 7: A schematic representation of the computational domain, consisting of two layers, the unknown seismic source (concentric circles), and the uniform array of receivers (black $\nabla$ ) placed at the ground surface. 

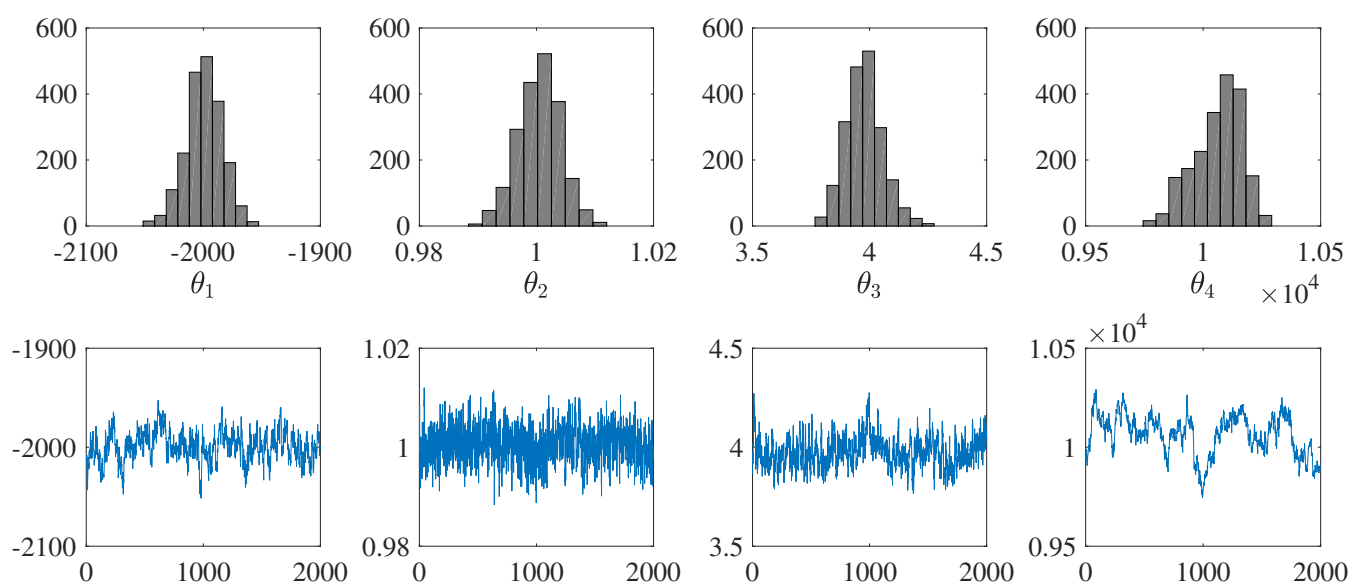

Figure 8: Posterior histograms and the trace plots of the Markov chain samples computed by the proposed Wasserstein-driven Bayesian technique.

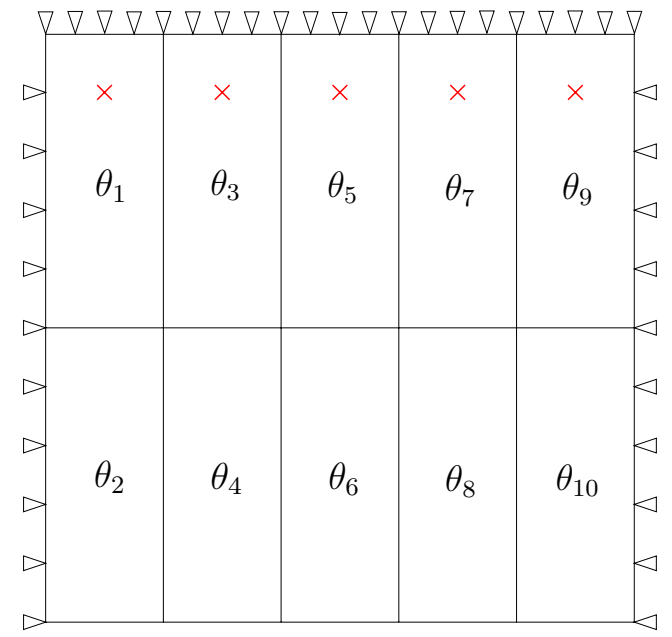

Figure 9: A schematic representation of the computational domain, the wave speed structure, and the arrays of sources $($ red $\times$ ) and the uniform array of receivers (black $\nabla$ ). 

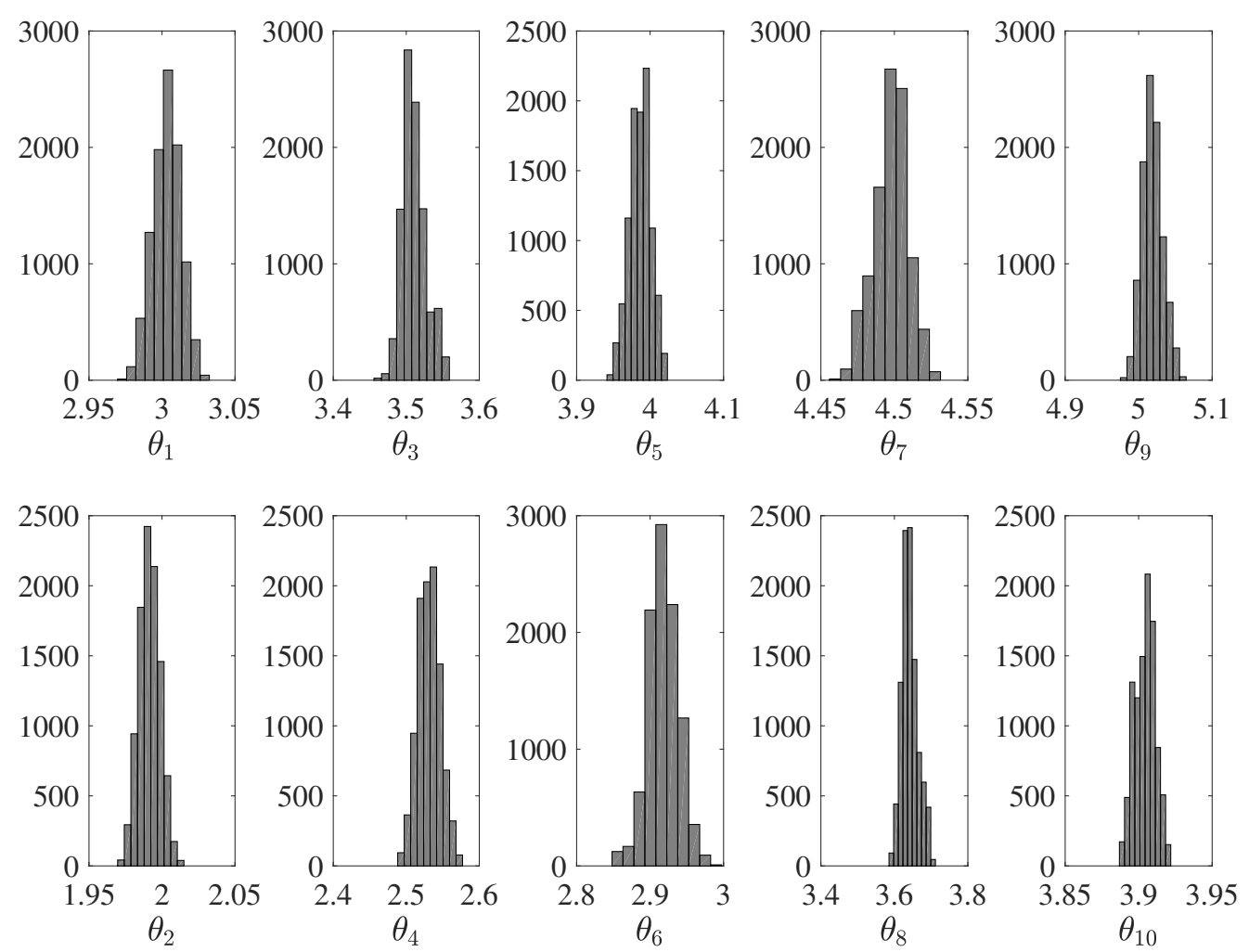

Figure 10: Posterior histograms of the Markov chain samples generated by the proposed Wasserstein-driven Bayesian algorithm.

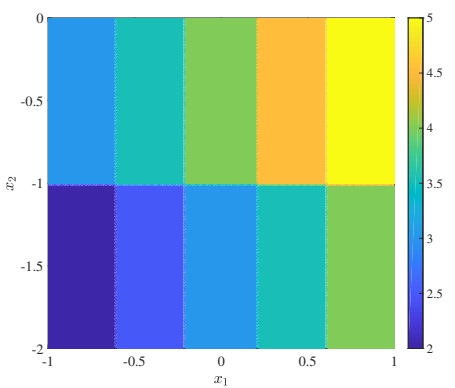

(a) $\boldsymbol{\theta}^{*}$

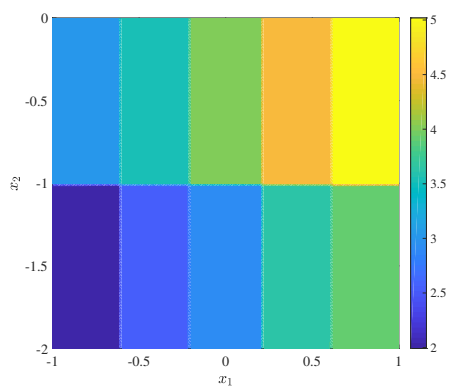

(b) $\mathbb{E}[\boldsymbol{\theta}]$

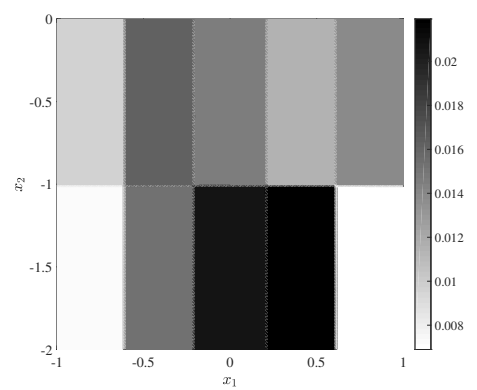

(c) $\mathbb{V}[\boldsymbol{\theta}]^{1 / 2}$

Figure 11: Map of the original noise-free parameters (a), the mean map (b) and the standard deviation map (c) obtained by the generated MCMC samples of the parameter posteriors. 